\title{
Mesoporous Silica Supported Au Nanoparticles with Controlled Size as Efficient Heterogeneous Catalyst for Aerobic Oxidation of Alcohols
}

\author{
Xuefeng Chu, ${ }^{1,2}$ Chao Wang, ${ }^{1}$ Liang Guo, ${ }^{1,2}$ Yaodan Chi, ${ }^{1}$ \\ Xiaohong Gao, ${ }^{1}$ and Xiaotian Yang ${ }^{1}$ \\ ${ }^{1}$ Jilin Provincial Key Laboratory of Architectural Electricity \& Comprehensive Energy Saving, \\ School of Electrical and Electronic Information Engineering, Jilin Jianzhu University, Changchun 130118, China \\ ${ }^{2}$ Department of Basic Science, Jilin Jianzhu University, Changchun 130118, China
}

Correspondence should be addressed to Xiaotian Yang; hanyxt@163.com

Received 24 August 2015; Revised 14 November 2015; Accepted 15 November 2015

Academic Editor: Jean-Luc Blin

Copyright (C) 2015 Xuefeng Chu et al. This is an open access article distributed under the Creative Commons Attribution License, which permits unrestricted use, distribution, and reproduction in any medium, provided the original work is properly cited.

A series of Au catalysts with different sizes were synthesized and employed on amine group functionalized ordered mesoporous silica solid supports as catalyst for the aerobic oxidation of various alcohols. The mesoporous silica of MCM-41 supported Au nanoparticles (Au-1) exhibited the smallest particle size at $\sim 1.8 \mathrm{~nm}$ with superior catalytic activities owing to the confinement effect of the mesoporous channels. Au-1 catalyst is also very stable and reusable under aerobic condition. Therefore, this presented work would obviously provide us a platform for synthesizing more size-controlled metal catalysts to improve the catalytic performances.

\section{Introduction}

Since the pioneering work of Hutchings and Haruta [1-7], Au nanoparticles were found to be an active material in catalytic science for a number of oxidation and hydrogenation reactions. Some recent work suggests that Au nanoparticles can be efficient catalysts for the selective oxidation of alcohols, olefins, and alkanes in the presence of molecular oxygen as oxidant $[1,2,8-15]$. The unique catalytic performance of $\mathrm{Au}$ nanoparticles is strongly depending upon the solid supports, particle size, shape, and some other factors [1, 2, 5-7, 16-23]. In many cases, $\mathrm{Au}$ nanoparticles size and the solid supports have a crucial role in enhancing the catalytic activity $[1,2,5-$ 23]. Furthermore, the junction between $\mathrm{Au}$ nanoparticles and metal oxide support contributes as active site for the catalytic reactions. Normally, Au nanoparticles supported on inert metal oxide supports (e.g., silica) result in relatively poor catalytic performances due to the lack of electronic interaction between the active sites and support [24, 25]. Therefore, the development of highly active Au nanoparticles on inert supports is very important for producing an efficient $\mathrm{Au}$ nanocatalyst.

The size dependent catalytic performance of Au nanoparticles has been extensively studied by previous reports, where the Au nanoparticle sizes were controlled by employing expensive ligand molecules with low loading efficiency on the solid supports in order to obtain highly active $\mathrm{Au}$ nanoparticles. For example, Wang and coworkers reported the pyrrolidone modified silica supported small-size $\mathrm{Au}$ nanoparticle $(\sim 4 \mathrm{~nm})$ is highly active for aerobic oxidation of alcohols [26]; Liu and coworkers found that the sub$2 \mathrm{~nm}$ Au nanoparticles synthesized using mercapto ligand are more active for the alkane oxidation than the larger ones [27]. More recently, Zhang and coworkers demonstrated that nanoporous ionic organic network could stabilize the smallsize Au nanoparticle at $\sim 2 \mathrm{~nm}$, giving high activity in aerobic oxidation of alcohols [28].

After the discovery of M41S family [29-33], ordered mesoporous silica (OMS) has emerged as an active material 
TABLE 1: Structural parameters and supported Au nanoparticles size on mesoporous silica with different amounts of Au loading.

\begin{tabular}{|c|c|c|c|c|c|}
\hline \multirow{2}{*}{ Sample } & \multirow{2}{*}{ Support } & \multirow{2}{*}{ Pore diameter (nm) } & \multirow{2}{*}{ Weight ratio of $\mathrm{Au}(\%)^{\mathrm{a}}$} & \multicolumn{2}{|c|}{ Au nanoparticles size (nm) } \\
\hline & & & & From XRD patterns ${ }^{\mathrm{b}}$ & From TEM images \\
\hline $\mathrm{Au}-1$ & MCM-41 & 3.0 & 2.4 & 1.4 & $1-3$ \\
\hline $\mathrm{Au}-2$ & SBA-15 & 8.5 & 3.0 & 2.3 & $2-4$ \\
\hline $\mathrm{Au}-3$ & SBA-15 & 8.5 & 9.1 & 4.2 & $5-9$ \\
\hline $\mathrm{Au}-4$ & MCM-41 & 3.0 & 10.3 & 5.4 & $5-15$ \\
\hline
\end{tabular}

${ }^{\mathrm{a}}$ The ratio of Au calculated from ICP.

${ }^{\mathrm{b}}$ The particle grain size calculated from Scherrer's equation.

to support metal nanoparticles for catalytic applications due to their high surface area, tunable and confined mesopores, diverse morphology, and large adsorption capacity. A significant amount of work has already been devoted to $\mathrm{Au}$ nanoparticles supported on mesoporous silica using variety of methods, but limited attention was only paid to the catalytic activity of size-controlled Au nanoparticles on OMS for aerobic oxidations $[34,35]$. In this work, we demonstrate an easy strategy to synthesize size-controlled Au nanoparticles as catalysts by using amine functionalized OMS solid support for aerobic oxidation of alcohols. MCM-41 and SBA-15 with same mesostructure $(p 6 \mathrm{~mm})$ but different pore sizes were considered as solid supports. The channel confinements of mesopores are utilized as template for the size-controlled synthesis of $\mathrm{Au}$ nanoparticles.

\section{Experimental Section}

2.1. Synthesis of Au Nanoparticles. MCM-41 and SBA-15 samples were prepared by using previously reported procedure. The samples were washed with the mixture of ethanol and $\mathrm{HCl}$ to remove the unreacted surfactants. $1 \mathrm{~g}$ of washed mesoporous silica was dried at $120^{\circ} \mathrm{C}$ under vacuum for 3 hours and then followed by the addition of $50 \mathrm{~mL}$ of pretreated toluene containing $1 \mathrm{~g}$ of DAPTS. The mixture was refluxed overnight. The experiments above were carried out using anhydrous condition to avoid any reaction between amine ligands and $\mathrm{H}_{2} \mathrm{O}$. Finally, the samples were collected by rotary evaporation and washed with a large quantity of ethanol for several times. $0.5 \mathrm{~g}$ of amine functionalized mesoporous samples was separately added into different volume of $\mathrm{HAuCl}_{4}$ solution (as shown in Table 1) and then stirred overnight. The product was collected and dried under vacuum for $1 \mathrm{~h}$ at $100^{\circ} \mathrm{C}$. Then, $\mathrm{NaBH}_{4}$ was used to reduce the gold(III) into gold nanoparticles as the following: $1 \mathrm{~g}$ of mesoporous silica containing gold(III) was added into $40 \mathrm{~mL}$ of anhydrous toluene and followed by the addition of $\mathrm{NaBH}_{4}$ under stirring. After 10 minutes of stirring, $10 \mathrm{~mL}$ of anhydrous ethanol was added into the mixture and stirred overnight. The products were collected by rotary evaporation and washed with a large quantity of ethanol and water for several times.

2.2. Characterization. X-ray diffraction (XRD) patterns were obtained with a Siemens D5005 diffractometer and Rigaku
D/MAX 2550 diffractometer using $\mathrm{CuK} \alpha$ radiation. The average grain size of gold nanoparticles was calculated by using half-width of the gold peak at $2 \theta=38.3^{\circ}$ by applying Scherrer's equation. Transmission electron microscopy (TEM) analysis was performed on a JEM-200CX electron microscope (JEOL, Japan) with an acceleration voltage of $300 \mathrm{kV}$. The nitrogen adsorption and desorption isotherms at the temperature of using liquid nitrogen were measured by using a Micromeritics ASAP Tristar system. The samples were outgassed for $10 \mathrm{~h}$ at $150^{\circ} \mathrm{C}$ before the measurements. The pore size distribution for mesoporous silica was calculated by using the Barrett-Joyner-Halenda (BJH) model. The contents of Au were determined by using inductively coupled plasma (ICP) with a Perkin-Elmer plasma 40 emission spectrometer. Diffuse reflectance ultraviolet-visible (UV-Vis) spectra were recorded on spectrometer of $\mathrm{PE}$ Lambda 20, and $\mathrm{BaSO}_{4}$ was an internal standard sample.

2.3. Catalytic Study. The catalytic study was performed on a $50 \mathrm{~mL}$ of glass reactor and the solution was stirred with a magnetic stirrer. The substrate and catalyst were mixed in the reactor and heated up to the given temperature. Then, oxygen was introduced into the reactor and sealed the reaction system. After $24 \mathrm{~h}$ reaction, the product was taken out from the system and analyzed by using gas chromatography (GC$17 \mathrm{~A}$, Shimadzu, using a flame ionization detector) with a flexible quartz capillary column coated with OV-17. The turnover frequencies (TOFs) were calculated based on the total number of $\mathrm{Au}$ atoms in the reaction system according to the following equation:

$$
\text { TOF }=\frac{\text { Converted mole of substrate }}{\text { Mole of Au atoms } * \text { Reaction time }} .
$$

\section{Results and Discussion}

3.1. Characterization of Au Nanoparticles Supported on Mesoporous Silica. XRD patterns of various Au nanoparticles supported on mesoporous silica samples are shown in Figure 1. The samples with higher amount of Au loading exhibited four well-resolved peaks in the range of $20-80^{\circ}$ as shown in Figure 1(a), indexed to the Au nanoparticles crystal planes of (111), (200), (220), and (311). However, the samples with lower amount of Au loading display a very broad peak at around $38.5^{\circ}$, suggesting decreased particle size compared 


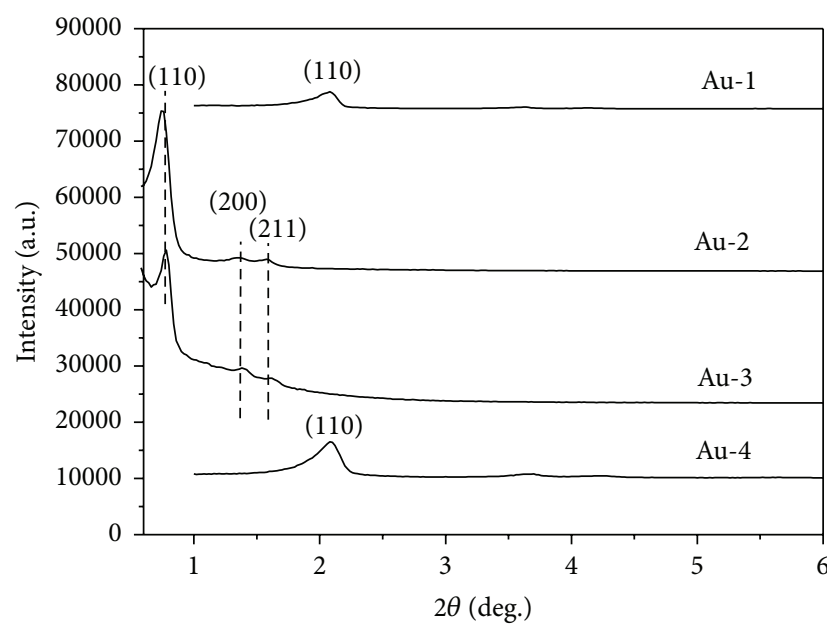

(a)

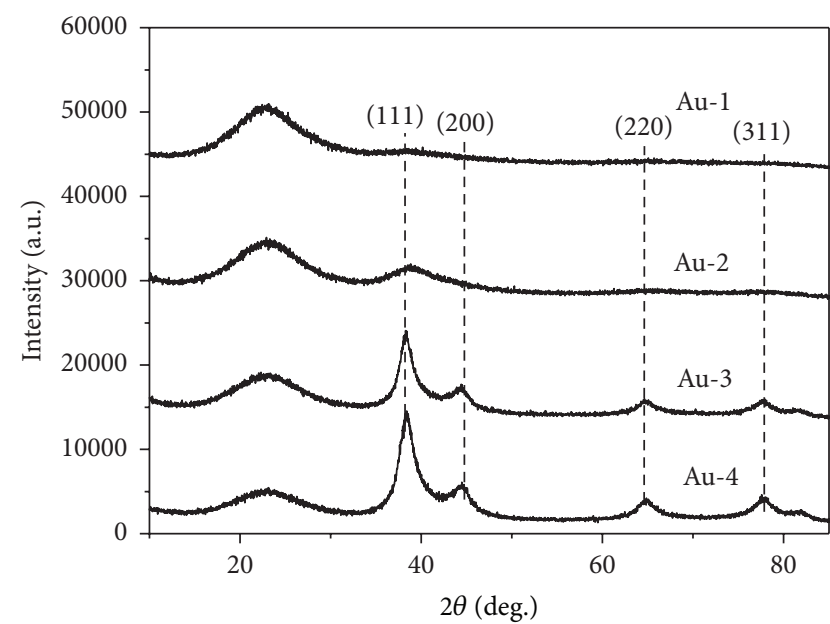

(b)

FIGURE 1: (a) Small- and (b) wide-angle XRD patterns of various Au nanoparticles supported mesoporous silica samples.

to the samples with higher Au loading. Additionally, the mesostructure of mesoporous silica remained stable during the Au loading process which confirmed with the low angle XRD patterns in Figure 1(b).

TEM analysis provides direct evidence for the $\mathrm{Au}$ nanoparticles size and the stable structure of the mesoporous silica (Figure 2). According to the TEM results, it is clear that the highly ordered structures along (110) or (100) crystal planes for 2D hexagonal mesoporous silica occurred in all samples. The well-dispersed Au nanoparticles within the mesoporous matrix except the sample of $\mathrm{Au}-4$ are shown in Figure 2(d). The size of Au nanoparticles was measured to be in the range 1-3 nm for the samples with low amount of $\mathrm{Au}$ loading as shown in Figures 2(a) and 2(b), which shows good agreement with the XRD patterns in Figure 1. Figure 2(c) shows TEM image for Au/SBA-15 with higher amount of Au loading, and the particle size distribution was centered in the range of 6-8 $\mathrm{nm}$ without any obvious aggregation. But an obvious aggregation occurred for Au nanoparticles supported on MCM-41 (Au-4) when higher amount of Au was loaded as shown in Figure 2(d) (Table 1).

Based on the results above, it can be confirmed that the $\mathrm{Au}$ nanoparticles $(2-3 \mathrm{~nm})$ were well dispersed in the mesoporous channels for both MCM-41 and SBA-15 when low amount of $\mathrm{Au}$ was loaded. In the case of higher amount of Au loading agglomeration occurred for MCM-41 as the pore diameter was relatively smaller at around $3 \mathrm{~nm}$, while SBA-15 has enough space to accommodate larger sized Au nanoparticles isolation with larger pore diameters of around $8.5 \mathrm{~nm}$.

3.2. Catalytic Properties of Au Nanoparticles for Aerobic Oxidation of Alcohols. 1-Phenylethanol and its paraderivates were chosen as model to investigate the catalytic properties of various $\mathrm{Au}$ catalysts. The obtained results were summarized in Table 2. Au-1 has showed highest catalytic conversion efficiency for all compounds and the turnover frequency
(TOF) was more than $300 \mathrm{~h}^{-1}$ which would be 3 times higher than that for efficient soluble catalysts of palladium complex [36]. The catalytic conversion efficiency of Au- 2 was also higher than $\mathrm{Au}-3$ and $\mathrm{Au}-4$ with higher amount of $\mathrm{Au}$ loading. The excellent catalytic performances of $\mathrm{Au}-1$ and $\mathrm{Au}-$ 2 should be attributed to the smaller Au nanoparticles size in the matrix of mesoporous silica. On the contrary, Au-4 showed very poor catalytic performance which might be due to the Au nanoparticles agglomeration. Even though there was no obvious aggregation for Au particles on SBA-15(H), the larger sized $\mathrm{Au}$ nanoparticles decreased the catalytic conversion.

3.3. Catalyst Stability. To investigate the stability of various Au catalysts, we recycled the samples for 5 times and calculated the catalytic conversion efficiency. Other than highest catalytic conversion efficiency, Au-1 catalyst is also highly stable and reusable as similar catalytic activity was observed while recycling the same sample for 5 times (Table 2). As shown in Figure 3, TEM images of Au-1, no changes were observed in the $\mathrm{Au}$ nanoparticles size after recycling the same sample for 5 times, which is totally different from the general phenomenon that the smaller particles would grow and become larger during the catalytic reactions. This is probably due to the limitation of mesopores of MCM-41, which can hinder the Au nanoparticles agglomeration during the catalytic reaction.

\section{Conclusion}

In conclusion, a series of Au catalysts with controlled sizes were synthesized on mesoporous silica solid supports. The MCM-41 supported Au nanocatalyst with lower amount of Au loading (Au-1) displayed higher catalytic activities than other catalysts for the aerobic oxidation of 1-phenylethanol and benzyl alcohol. The Au-1 catalyst also showed highly stable and reusable capability while recycling the same sample 

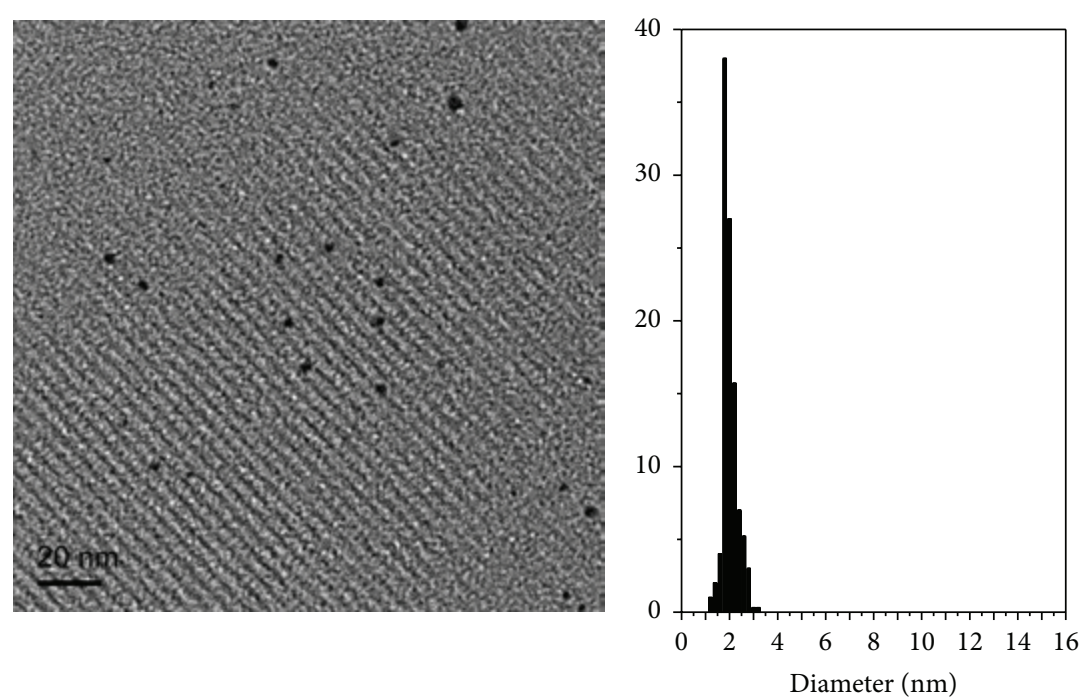

(a)
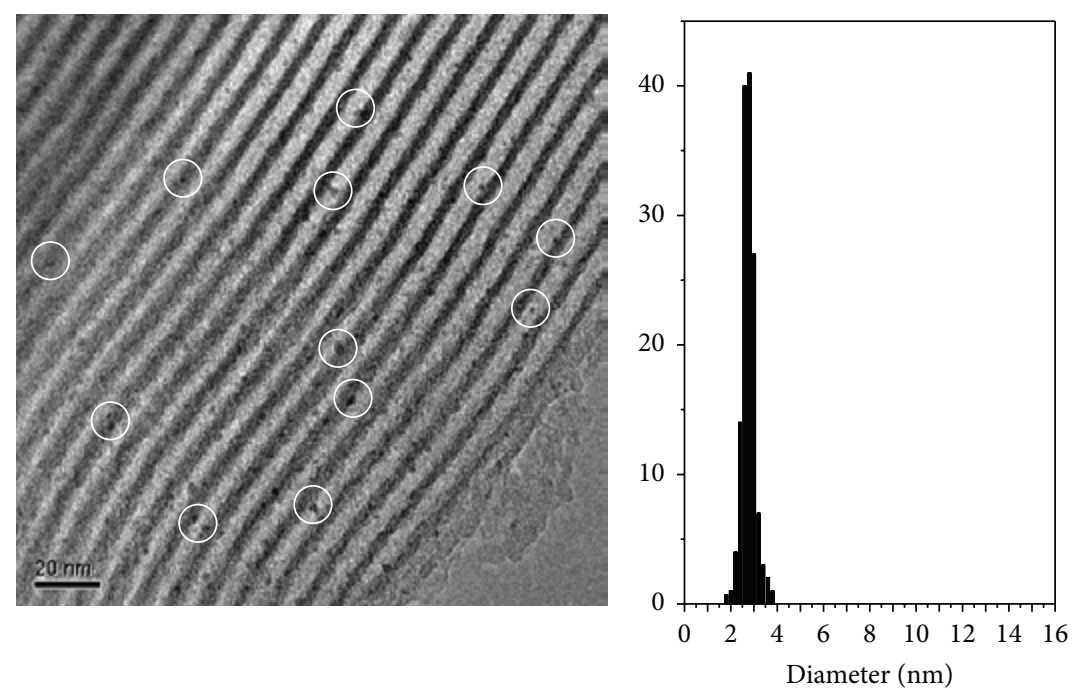

(b)
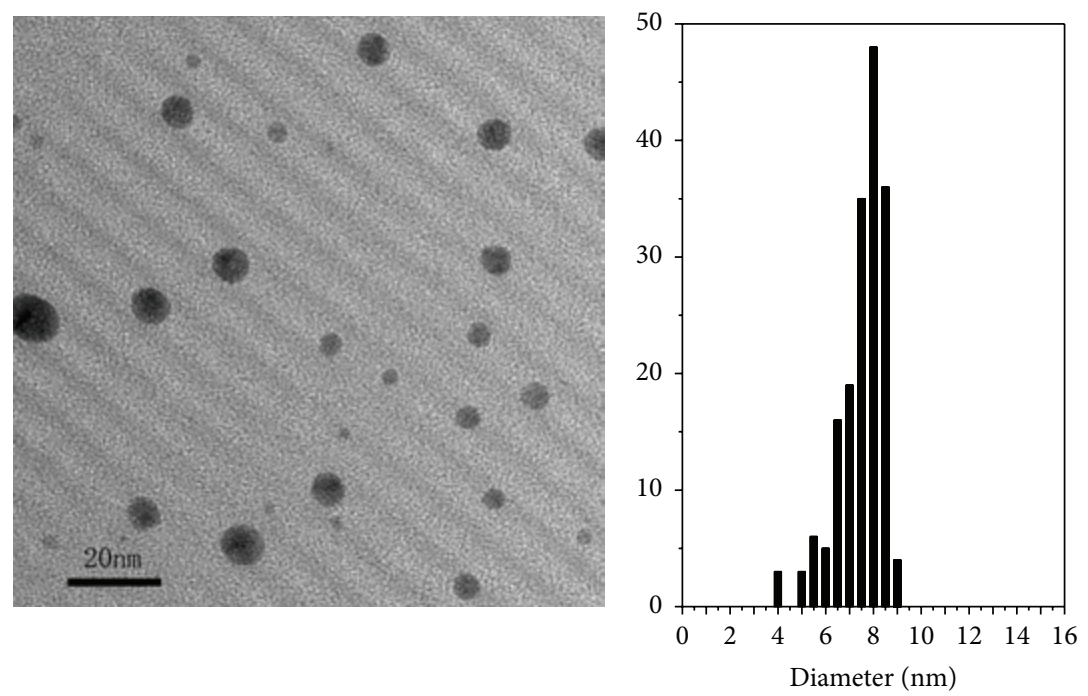

(c)

Figure 2: Continued. 

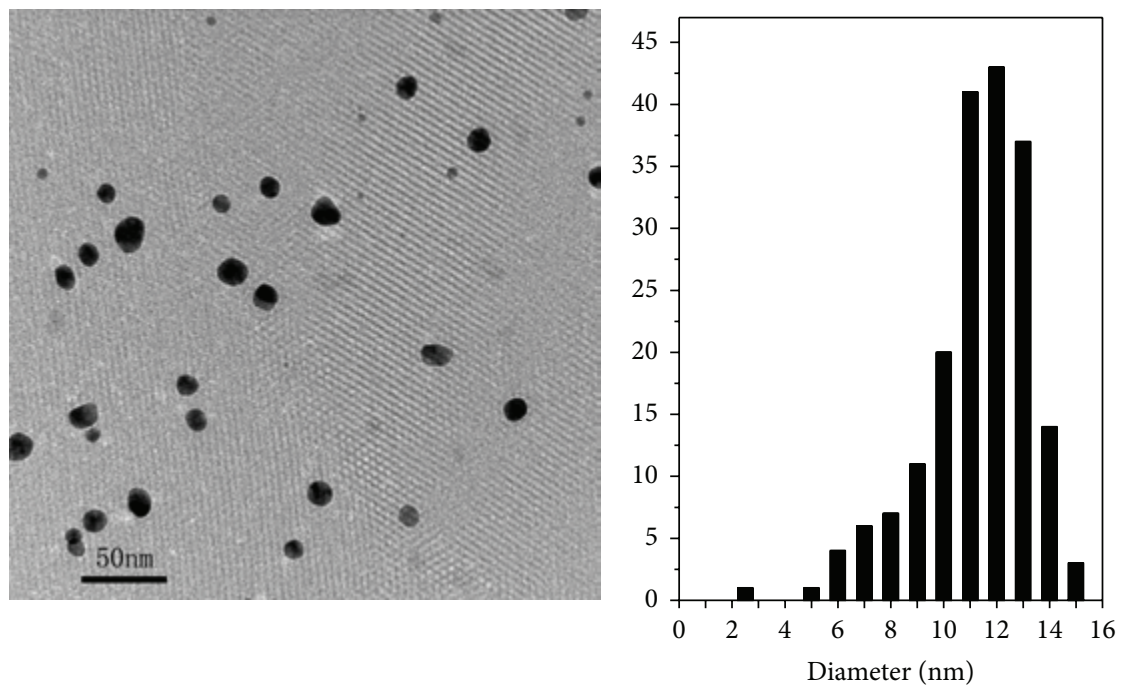

(d)

Figure 2: TEM images and size distributions for (a) Au-1, (b) Au-2, (c) Au-3, and (d) Au-4 catalysts.

TABLE 2: Catalytic activities of various Au/OMS samples for aerobic oxidations of 1-phenylethanol and its paraderivates ${ }^{\mathrm{a}}$.

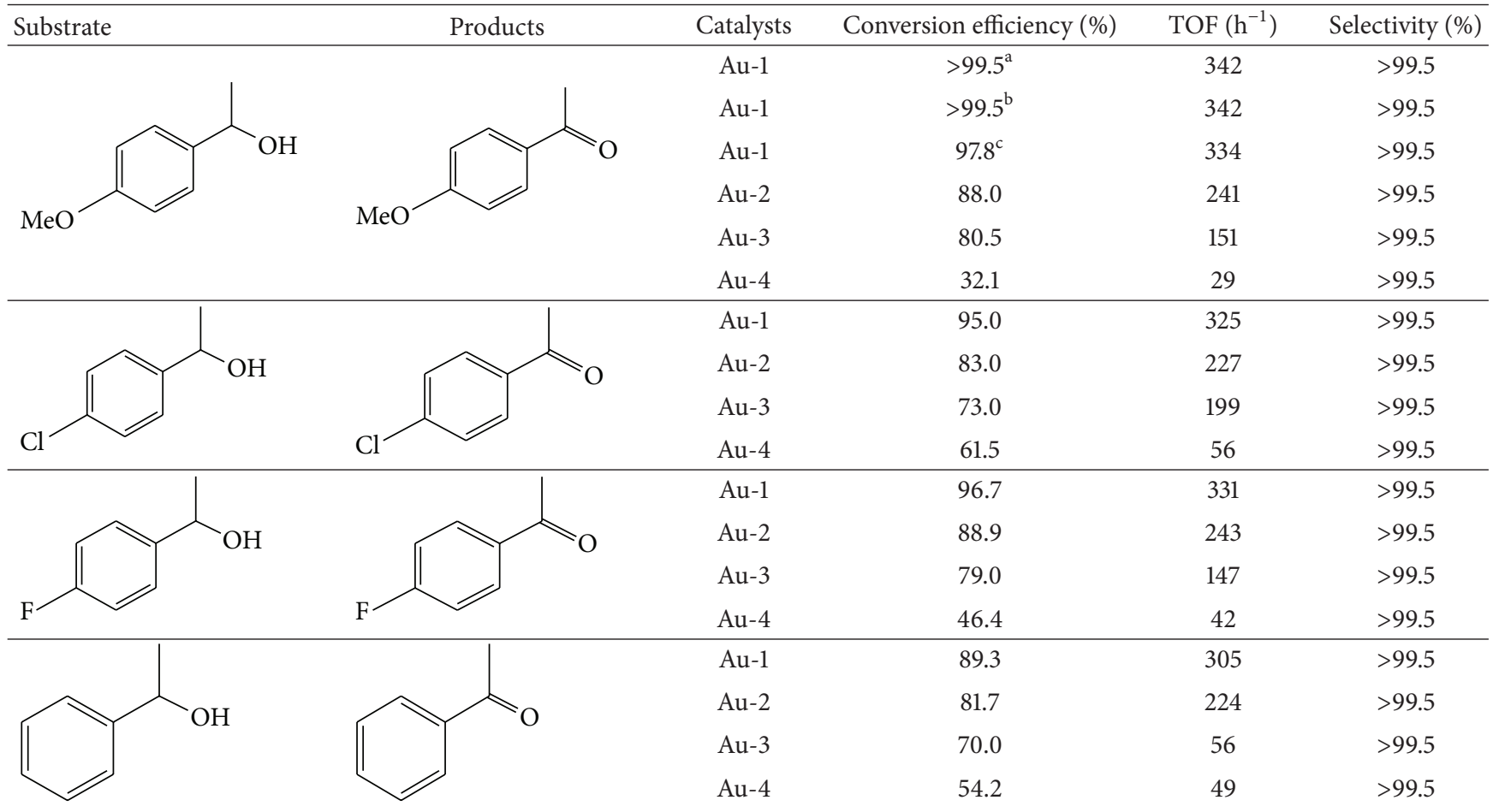

${ }^{\mathrm{a}}$ Reactions conditions: $60 \mathrm{mg}$ of catalyst, $0.6 \mathrm{mmol}$ of substrates, $0.3 \mathrm{mmol}$ of $\mathrm{Na}_{2} \mathrm{CO}_{3}, 100^{\circ} \mathrm{C}, 24 \mathrm{~h}$, and $6 \mathrm{~mL}$ of toluene as solvent.

${ }^{\mathrm{b}}$ Recycled results.

${ }^{\mathrm{c}}$ Recycled for 5 times. The sample was dried at $140^{\circ} \mathrm{C}$ before the 5 th recycle.

for 5 times. Therefore, this work would definitely provide a platform for the future about synthesizing more active metal nanoparticles on inert solid supports for the efficient catalytic conversion.

\section{Conflict of Interests}

The authors declare that there is no conflict of interests regarding the publication of this paper. 


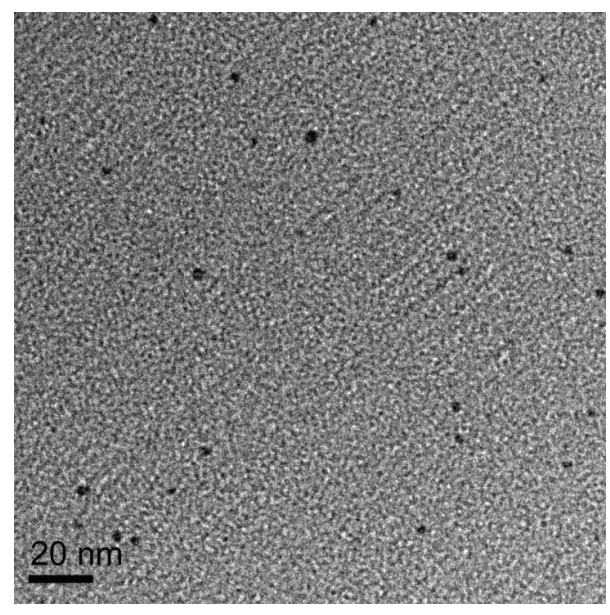

FIgURE 3: TEM images of Au-1 catalyst after recycling for 5 times.

\section{Acknowledgments}

The authors gratefully acknowledge the support from Natural Science Foundation of Jilin Province (nos. 20110323, 20121822, 20140520080JH, and 20140520120JH) and Natural Science Foundation of Jilin Province, Department of Education (no. 2014238).

\section{References}

[1] A. S. K. Hashmi and G. J. Hutchings, "Gold catalysis," Angewandte Chemie - International Edition, vol. 45, no. 47, pp. 78967936, 2006

[2] G. J. Hutchings, "A golden future for green chemistry," Catalysis Today, vol. 122, no. 3-4, pp. 196-200, 2007.

[3] G. J. Hutchings, "Vapor phase hydrochlorination of acetylene: correlation of catalytic activity of supported metal chloride catalysts," Journal of Catalysis, vol. 96, no. 1, pp. 292-295, 1985.

[4] M. Haruta, T. Koboyashi, H. Sano, and N. Yamada, "Novel gold catalysts for the oxidation of carbon monoxide at a temperature far below $0^{\circ}$ C," Chemical Letters, vol. 10, pp. 405-408, 1987.

[5] T. V. Choudhary and D. W. Goodman, "Oxidation catalysis by supported gold nano-clusters," Topics in Catalysis, vol. 21, no. 1, pp. 25-34, 2002.

[6] M. Chen and D. W. Goodman, "Catalytically active gold: from nanoparticles to ultrathin films," Accounts of Chemical Research, vol. 39, no. 10, pp. 739-746, 2006.

[7] M. Chen and D. W. Goodman, "Catalytically active gold on ordered titania supports," Chemical Society Reviews, vol. 37, no. 9, pp. 1860-1870, 2008.

[8] A. Abad, P. Concepcion, A. Corma, and H. Garcia, "A collaborative effect between gold and a support induces the selective oxidation of alcohols," Angewandte Chemie International Edition, vol. 44, no. 26, pp. 4066-4069, 2005.

[9] L. Chen, J. Hu, and R. Richards, "Intercalation of aggregationfree and well-dispersed gold nanoparticles into the walls of mesoporous silica as a robust 'green' catalyst for $n$-alkane oxidation," Journal of the American Chemical Society, vol. 131, no. 3, pp. 914-915, 2009.

[10] A. Grirrane, A. Corma, and H. Garcia, "Highly active and selective gold catalysts for the aerobic oxidative condensation of benzylamines to imines and one-pot, two-step synthesis of secondary benzylamines," Journal of Catalysis, vol. 264, no. 2, pp. 138-144, 2009.

[11] A. Grirrane, A. Corma, and H. Garcia, "Gold nanoparticles supported on ceria promote the selective oxidation of oximes into the corresponding carbonylic compounds," Journal of Catalysis, vol. 268, no. 2, pp. 350-355, 2009.

[12] A. Grirrane, A. Corma, and H. García, "Gold-catalyzed synthesis of aromatic azo compounds from anilines and nitroaromatics," Science, vol. 322, no. 5908, pp. 1661-1664, 2008.

[13] M. Turner, V. B. Golovko, O. P. H. Vaughan et al., "Selective oxidation with dioxygen by gold nanoparticle catalysts derived from 55-atom clusters," Nature, vol. 454, no. 7207, pp. 981-983, 2008.

[14] M. D. Hughes, Y.-J. Xu, P. Jenkins et al., "Tunable gold catalysts for selective hydrocarbon oxidation under mild conditions," Nature, vol. 437, no. 7062, pp. 1132-1135, 2005.

[15] A. Corma and H. Garcia, "Supported gold nanoparticles as catalysts for organic reactions," Chemical Society Reviews, vol. 37, no. 9, pp. 2096-2126, 2008.

[16] A. A. Herzing, C. J. Kiely, A. F. Carley, P. Landon, and G. J. Hutchings, "Identification of active gold nanoclusters on iron oxide supports for CO oxidation," Science, vol. 321, no. 5894, pp. 1331-1335, 2008.

[17] D. I. Rnache, D. W. Knight, and G. J. Hutchings, "Solvent-free oxidation of primary alcohols to aldehydes using supported gold catalysts," Catalysis Letters, vol. 103, no. 1, pp. 43-52, 2005.

[18] C. Raptis, H. Garcia, and M. Stratakis, "Selective isomerization of epoxides to allylic alcohols catalyzed by $\mathrm{TiO}_{2}$-supported gold nanoparticles," Angewandte Chemie-International Edition, vol. 48, no. 17, pp. 3133-3136, 2009.

[19] J. J. Bravo-Suárez, K. K. Bando, J. Lu, T. Fujitani, and S. T. Oyama, "Oxidation of propane to propylene oxide on gold catalysts," Journal of Catalysis, vol. 255, no. 1, pp. 114-126, 2008.

[20] H. Sun, F. Su, J. Ni, Y. Cao, H. He, and K. Fan, "Gold supported on hydroxyapatite as a versatile multifunctional catalyst for the direct tandem synthesis of imines and oximes," Angewandte Chemie-International Edition, vol. 48, no. 24, pp. 4390-4393, 2009.

[21] F. Su, Y. Liu, L. Wang, Y. Cao, H. He, and K. Fan, "Ga-Al mixedoxide-supported gold nanoparticles with enhanced activity for aerobic alcohol oxidation," Angewandte Chemie-International Edition, vol. 47, no. 2, pp. 334-337, 2007.

[22] S. Biella and M. Rossi, "Gas phase oxidation of alcohols to aldehydes or ketones catalysed by supported gold," Chemical Communications, no. 3, pp. 378-379, 2003.

[23] S. Biella, L. Prati, and M. Rossi, "Selective oxidation of Dglucose on gold catalyst," Journal of Catalysis, vol. 206, no. 2, pp. 242-247, 2002.

[24] K. Okazaki, Y. Morikawa, S. Tanaka, K. Tanaka, and M. Kohyama, "Electronic structures of $\mathrm{Au}$ on $\mathrm{TiO}_{2}(110)$ by firstprinciples calculations," Physical Review B-Condensed Matter and Materials Physics, vol. 69, no. 23, Article ID 235404, 2004.

[25] K. Okazaki, S. Ichikawa, Y. Maeda, M. Haruta, and M. Kohyama, "Electronic structures of $\mathrm{Au}$ supported on $\mathrm{TiO}_{2}$," Applied Catalysis A: General, vol. 291, no. 1-2, pp. 45-54, 2005.

[26] L. Wang, X. Meng, B. Wang, W. Chi, and F.-S. Xiao, "Pyrrolidone-modified SBA-15 supported Au nanoparticles with superior catalytic properties in aerobic oxidation of alcohols," Chemical Communications, vol. 46, no. 27, pp. 5003-5005, 2010. 
[27] Y. Liu, H. Tsunoyama, T. Akita, S. Xie, and T. Tsukuda, "Aerobic oxidation of cyclohexane catalyzed by size-controlled au clusters on hydroxyapatite: size effect in the sub- $2 \mathrm{~nm}$ regime," ACS Catalysis, vol. 1, no. 1, pp. 2-6, 2011.

[28] P. Zhang, Z.-A. Qiao, X. Jiang, G. M. Veith, and S. Dai, "Nanoporous ionic organic networks: stabilizing and supporting gold nanoparticles for catalysis," Nano Letters, vol. 15, no. 2, pp. 823-828, 2015.

[29] C. T. Kresge, M. E. Leonowicz, W. J. Roth, J. C. Vartuli, and J. S. Beck, "Ordered mesoporous molecular sieves synthesized by a liquid-crystal template mechanism," Nature, vol. 359, no. 6397, pp. 710-712, 1992.

[30] J. S. Beck, J. C. Vartuli, W. J. Roth et al., "A new family of mesoporous molecular sieves prepared with liquid crystal templates," Journal of the American Chemical Society, vol. 114, no. 27, pp. 10834-10843, 1992.

[31] D. Zhao, J. Feng, Q. Huo et al., "Triblock copolymer syntheses of mesoporous silica with periodic 50 to 300 angstrom pores," Science, vol. 279, no. 5350, pp. 548-552, 1998.

[32] D. Zhao, Q. Huo, J. Feng, B. F. Chmelka, and G. D. Stucky, "Nonionic triblock and star diblock copolymer and oligomeric surfactant syntheses of highly ordered, hydrothermally stable, mesoporous silica structures," Journal of the American Chemical Society, vol. 120, no. 24, pp. 6024-6036, 1998.

[33] Y. Wan and D. Zhao, "On the controllable soft-templating approach to mesoporous silicates," Chemical Reviews, vol. 107, no. 7, pp. 2821-2860, 2007.

[34] H. Li, Z. Zheng, M. Cao, and R. Cao, "Stable gold nanoparticle encapsulated in silica-dendrimers organic-inorganic hybrid composite as recyclable catalyst for oxidation of alcohol," Microporous and Mesoporous Materials, vol. 136, no. 1-3, pp. 4249, 2010.

[35] S. Kim, S. W. Bae, J. S. Lee, and J. Parka, "Recyclable gold nanoparticle catalyst for the aerobic alcohol oxidation and $\mathrm{C}-\mathrm{C}$ bond forming reaction between primary alcohols and ketones under ambient conditions," Tetrahedron, vol. 65, no. 7, pp. 14611466, 2009.

[36] G.-J. ten Brink, I. W. C. E. Arends, and R. A. Sheldon, "Green, catalytic oxidation of alcohols in water," Science, vol. 287 , no. 5458, pp. 1636-1639, 2000. 

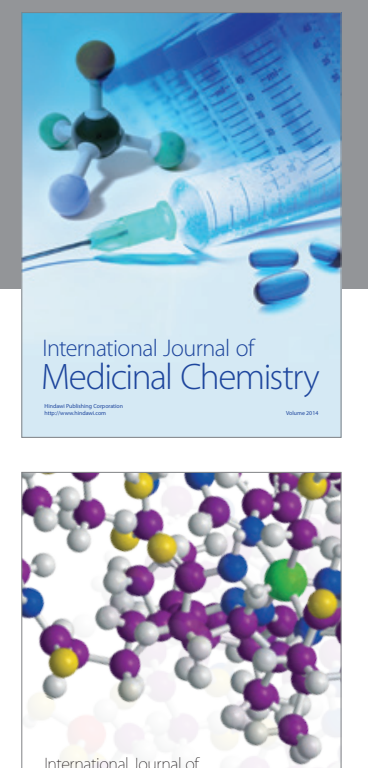

\section{Carbohydrate} Chemistry

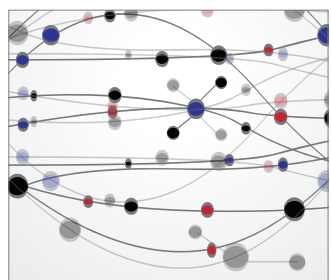

The Scientific World Journal
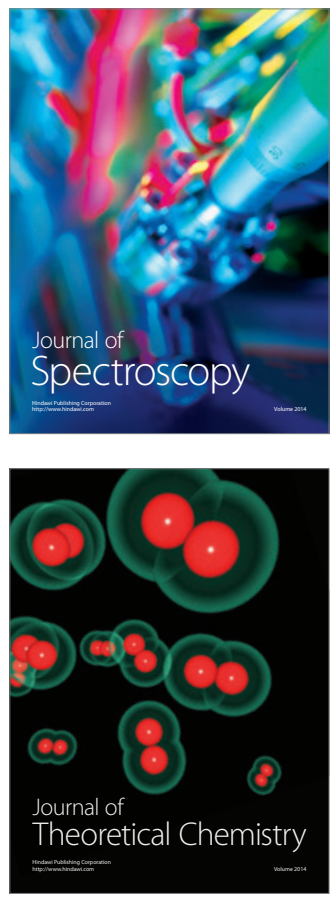
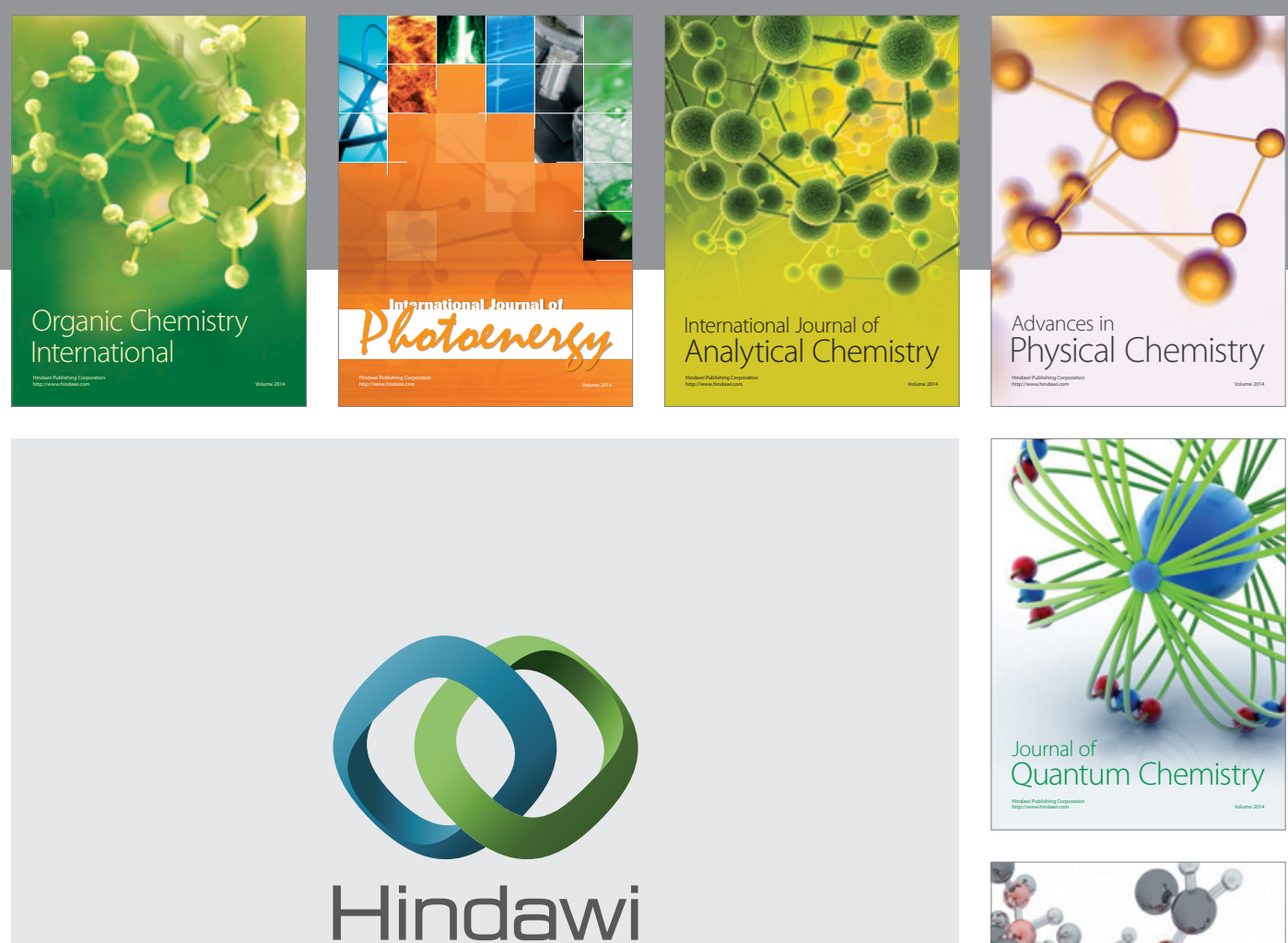

Submit your manuscripts at

http://www.hindawi.com

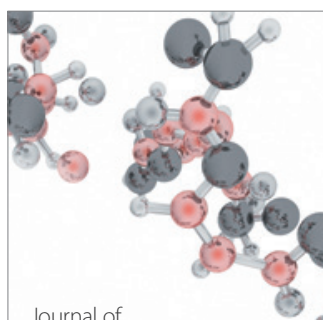

Analytical Methods

in Chemistry

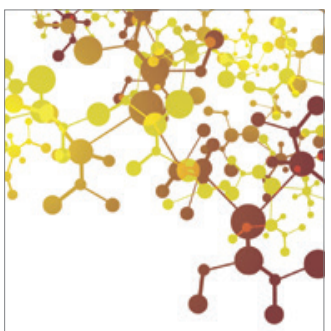

Journal of

Applied Chemistry

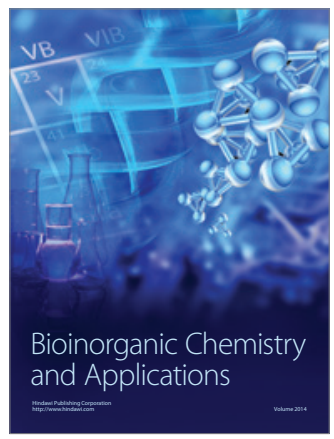

Inorganic Chemistry
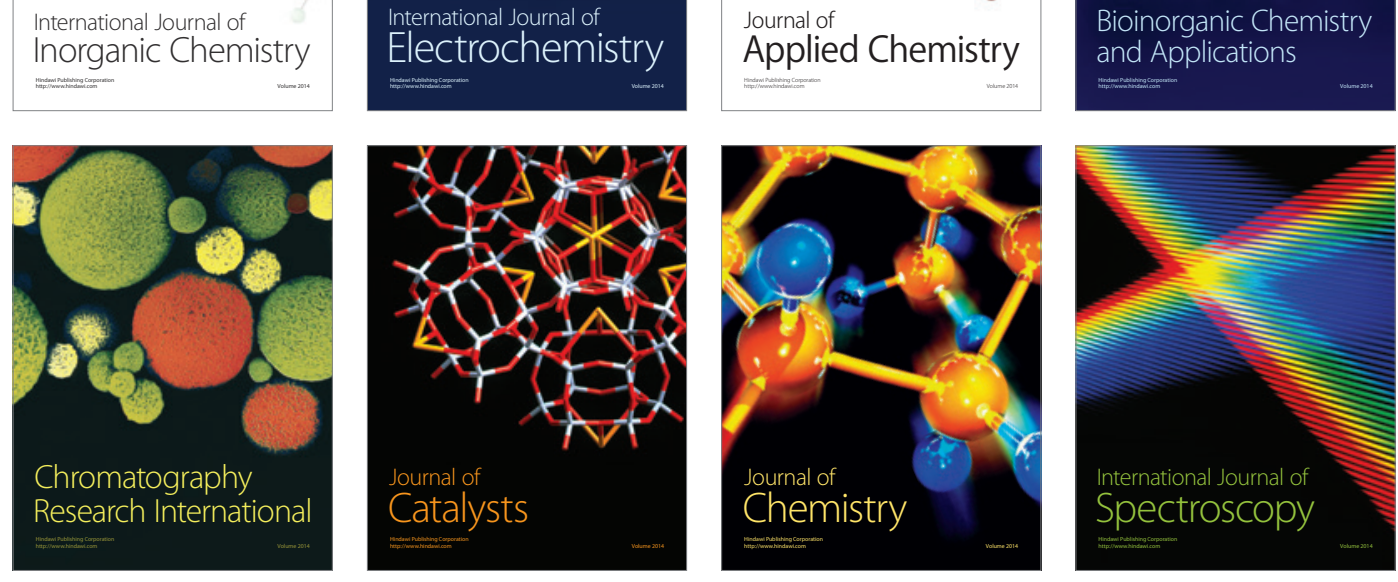